\title{
GRAPHS OF TOTALLY GEODESIC FOLIATIONS ON PSEUDO-RIEMANNIAN MANIFOLDS
}

\author{
N.I. ZHUKOVA
}

\begin{abstract}
We study totally geodesic foliations $(M, F)$ of arbitrary codimension $q$ on $n$-dimensional pseudo-Riemannian manifolds, for which the induced metrics on leaves is non-degenerate. We assume that the $q$-dimensional orthogonal distribution $\mathfrak{M}$ to $(M, F)$ is an Ehresmann connection for this foliation. Since the usual graph $G(F)$ is not Hausdorff manifold in general, we study the graph $G_{\mathfrak{M}}(F)$ of the foliation with an Ehresmann connection $\mathfrak{M}$ introduced early by the author. This graph is always a Hausdorff manifold. We prove that on the graph $G_{\mathfrak{M}}(F)$, a pseudo-Riemannian metric is defined, with respect to which the induced foliation and the simple foliations formed by the leaves of the canonical projections are totally geodesic. We show that the leaves of the induced foliation on the graph are non-degenerately reducible pseudo-Riemannian manifolds and their structure is described. The application to parallel foliations on nondegenerately reducible pseudo-Riemannian manifolds is considered. We also show that each foliation defined by the suspension of a homomorphism of the fundamental group of a pseudo-Riemannian manifold belongs to the considered class of foliations.
\end{abstract}

Keywords: totally geodesic foliation, pseudo-Riemaniann manifold, graph of a foliation, Ehresmann connection for a foliation.

Mathematics Subject Classification: 53C12, 53C50, 57R30

\section{INTRODUCTION AND MAIN RESULTS}

Foliation golonomy gruppoid was introduced by Ch. Ehresmann and an equivalent construction was proposed by H.E. Winkelnkemper [1] and was called the graph of a foliation. The graph of a foliation contains all information about the foliation and on germ holonomy groups conventionally accepted in the foliation theory [2]. $C^{*}$-algebras of complex-valued functions for foliations introduced by Connes [3] are defined on the holonomy gruppoids of these foliations.

Let $(M, F)$ be a smooth foliation of codimension $q$ on an $n$-dimensional smooth manifold $M$. R. Blumenthal and J. Hebda defined an Ehresmann connection for $(M, F)$ as a $q$-dimensional distribution $\mathfrak{M}$ transversal to the leaves whose integral curves admit a translation along each curve in a leaf of the foliation [4], 5]. For an arbitrary leaf $L_{\alpha}$ of the foliation $(M, F)$, the notion of a $\mathfrak{M}$-holonomy group was introduced. We provide exact definitions in Section 2.

Let us recall the construction of the graph $G_{\mathfrak{M}}(F)$ of a foliation $F$ of a codimension $q$ with an Ehresmann connection $\mathfrak{M}$ on an $n$-dimensional manifold $M$ introduced by us [6], see also [7, [8]. Let us consider two points $x$ and $y$ in the same leaf $L_{\alpha}$ in this foliation, We denote by $A(x, y)$ the set of all piecewise smooth paths in $L_{\alpha}$ connecting $x$ and $y$. Paths $h$ and $f$ in $A(x, y)$ are called equivalent $h \sim f$ if the loop $h \cdot f^{-1}$ equalling to the product of the paths $h$ and $f^{-1}$ generates a trivial element of a $\mathfrak{M}$-holonomy group $H_{\mathfrak{M}}\left(L_{\alpha}, x\right)$ of a leaf $L_{\alpha}$ at a point $x$. The equivalence class containing a path $h$ is denoted by $\{h\}$. The set $G_{\mathfrak{M}}(F)$ of triples of form $(x,\{h\}, y)$, where $x \in M$, $y \in L(x), h \in A(x, y)$, is called the graph of the foliation $(M, F)$ with an Ehresmann connection $\mathfrak{M}$, while the mappings $p_{1}: G_{\mathfrak{M}}(F) \rightarrow M:(x,\{h\}, y) \mapsto x, p_{2}: G_{\mathfrak{M}}(F) \rightarrow M:(x,\{h\}, y) \mapsto y$ are called

N.I. Zhukova, Graphs of totally GEODESic FOLIATIONS ON PSEUdo-RIEMANNiAn MANifolds.

(c)Zhukova N.I. 2019.

The work is supported by RFBR (grant no. 16-11-00312) and by the Center of Fundamental Research of HSE in 2019.

Submitted July 19, 2018. 
the canonical projections. We show that the graph $G_{\mathfrak{M}}(F)$ is naturally equipped with a structure of $(2 n-q)$-dimensional smooth Hausdorff manifold [6], see also [7], 8].

Thus, the graph of a foliation with an Ehresmann connection $G_{\mathfrak{M}}(F)$ is defined similar to a classical graph of a foliation $G(F)$ [1] by replacing the germ holonomy group $\Gamma(L, x)$ of a leaf $L, x \in M$, by the $\mathfrak{M}$-holonomy group $H_{\mathfrak{M}}(L, x)$. In distinction to $G_{\mathfrak{M}}(F)$, a topological space of the graph $G(F)$ is generally speaking not Hausdorff.

The mapping

$$
\beta: G_{\mathfrak{M}}(F) \rightarrow G(F), \beta(x,\{h\}, y)=(x,<h>, y),
$$

where $(x,<h>, y) \in G(F)$, is a local diffeomorphism. Both graphs $G(F)$ and $G_{\mathfrak{M}}(F)$ are equipped with the structure of gruppoids and $\beta$ is a homomorphism of these gruppoids, that is, it maps the product of the elements in one gruppoid into that of corresponding elements in another grouppoid.

Let $p: M \rightarrow B$ be a submersion and $\mathfrak{M}$ be a distribution on $B$. We shall employ the notation $p^{*} \mathfrak{M}:=\left\{\mathfrak{N}_{x} \mid x \in M\right\}$, where $\mathfrak{N}_{x}=\left\{Y \in T_{x} M \mid p_{* x} Y \in \mathfrak{M}_{p(x)}\right\}$

We proved the following properties of the two aforementioned graphs of an arbitrary foliation with an Ehresmann connection [6], 7].

Theorem 1. Let $(M, F)$ be a foliation of codimension $q$ on a smooth n-dimensional manifold $M$ admitting an Ehresmann connection $\mathfrak{M}$. Then

1. A homomorphism $\beta: G_{\mathfrak{M}}(F) \rightarrow G(F)$ of gruppoids is an isomorphism if and only if the graph $G(F)$ is Hausdorff.

2. The canonical projections $p_{i}: G_{\mathfrak{M}}(F) \rightarrow M, i=1,2$, are locally trivial fibrations.

3. The distribution $\mathfrak{N}:=p_{1}^{*} \mathfrak{M} \cap p_{2}^{*} \mathfrak{M}$ is an Ehresmann connection for the induced foliation

$$
\mathbb{F}:=\left\{p_{1}^{-1}(L) \mid L \in F\right\}=\left\{p_{2}^{-1}(L) \mid L \in F\right\},
$$

on the graph $G_{\mathfrak{M}}(F)$, and the holonomy groups $H_{\mathfrak{N}}(\mathbb{L}, z)$ and $H_{\mathfrak{M}}(L, x), x=p_{1}(z)$ of leaves $\mathbb{L}$ and $L=p_{1}(\mathbb{L})$ and their germ holonomy groups are canonically isomorphic.

Definition 1. A pseudogroup $\mathcal{H}$ of locally holonomic diffeomorphisms of a manifold $M$ is called quasianalytic if the fact that for some open connected subset $V$ in $M$ the identity $h_{V}=i d_{V}$ holds for some element $h \in \mathcal{H}$ implies that $h=i d_{D(h)}$ on the entire connected domain $D(h)$ of the element $h$ containing $V$.

According [9, Prop. 2], the Winkelnkemper critetion on the Hausdorff property of the graph $G(F)$ can be reformulated as follows.

Proposition 1. A topological space of the graph $G(F)$ of the foloation $(M, F)$ is Hausdorff if and only the holonomy pseudogroup of this foliation is quasianalytic.

According Theorem 1 and Proposition 1 for the foliations with an Ehresmann connection having a quasianalytic holonomy pseudogroup, we can identify the graphs $G_{\mathfrak{M}}(F)$ and $G(F)$ by the canonical isomorphism $\beta$ and we denote this by $G_{\mathfrak{M}}(F) \cong G(F)$. Therefore, the graph $G_{\mathfrak{M}}(F)$ can be considered as a desingularization of a non-Hausdorff graph $G(F)$, where by the singularity we mean the absence of the Hausdorff property. Such essential difference between the properties of these graphs is explained by the fact that the $\mathfrak{M}$-holonomy group $H_{\mathfrak{M}}\left(L_{\alpha}, x\right)$ is of a global nature, while germ holonomy group $\Gamma\left(L_{\alpha}, x\right)$ has a local-global nature; the global one is over the leaves and the local one is over the transversals.

The following three foliations are induced on the graph $G_{\mathfrak{M}}(F)$ :

$$
F^{(1)}=\left\{p_{1}^{-1}(x) \mid x \in M\right\}, \quad F^{(2)}=\left\{p_{2}^{-1}(x) \mid x \in M\right\}, \quad \mathbb{F}=\left\{p_{1}^{-1}(L) \mid L \in F\right\} .
$$

We observe $\mathbb{F}=\left\{p_{2}^{-1}(L) \mid L \in F\right\}$.

We introduce the notation $\mathfrak{N}=p_{1}^{*} \mathfrak{M} \cap p_{2}^{*} \mathfrak{M}$ and $\mathfrak{M}^{(1)}=\mathfrak{N} \oplus T F^{(2)}$. We stress that each smooth vector field $X$ on the graph $G_{\mathfrak{M}}(F)$ is uniquely represented as $X=X^{(1)}+X^{\mathfrak{N}}+X^{(2)}$, where $X^{(i)} \in$ $\mathfrak{X}_{F^{(i)}}\left(G_{\mathfrak{M}}(F)\right), i=1,2, X^{\mathfrak{N}} \in \mathfrak{X}_{\mathfrak{N}}\left(G_{\mathfrak{M}}(F)\right)$, as well as in the form:

$$
X=X^{(1)}+X^{\mathfrak{M}^{(1)}},
$$

where $X^{\mathfrak{M}^{(1)}} \in \mathfrak{X}_{\mathfrak{M}^{(1)}}\left(G_{\mathfrak{M}}(F)\right)$. 
Definition 2. Let $(M, F)$ be a foliation of a codimension $q$ on n-dimensional pseudo-Riemannian manifold $(M, g), 0<q<n$, and on the leaves pseudo-Riemannian metrics are induced. Then for all vector fields $X, Y \in \mathfrak{X}\left(G_{\mathfrak{M}}(F)\right)$ given by (1) the identity

$$
d(X, Y):=\left(p_{1}^{*} g\right)\left(X^{\mathfrak{M}^{(1)}}, Y^{\mathfrak{M}^{(1)}}\right)+\left(p_{2}^{*} g\right)\left(X^{(1)}, Y^{(1)}\right)
$$

defines a pseudo-Riemannian metrics $d$ on the graph $G_{\mathfrak{M}}(F)$ called the induced metrics.

As $(M, F)$ is a transversally analytic Riemannian foliation and $\mathfrak{M}$ is the $q$-dimensional orthogonal distribution on a complete Riemannian manifold and the graph $G(F)$ is identified with the graph $G_{\mathfrak{M}}(F)$, the induced metrics $d$ on $G(F)$ was considered by R. Wolak in [10].

Definition 3. A distribution $\mathfrak{M}$ on a pseudo-Riemannian manifold $(M, g)$ is called geodesically invariant if each smooth geodesic curve of the Levi-Civita connection of the metrics $g$ touching the distribution $\mathfrak{M}$ at a single point is an integral curve of this distribution.

Foliations with a geodesically invariant tangential distribution are called the totally geodesic foliations.

A following theorem is one of the main results of the present work.

Theorem 2. Let $(M, F)$ be a totally geodesic foliation of an arbitrary codimension $q$ on an $n$ dimensional pseudo-Riemannian manifold $(M, g)$, and on the leaves, pseudo-Riemannian metrics are induced. Assume that a q-dimensional distribution $\mathfrak{M}$ orthogonal to the foliation $(M, F)$ is an Ehresmann connection for $(M, F)$. Then the above defined foliations $F^{(1)}, F^{(2)}$ and $\mathbb{F}$ on the graph $G_{\mathfrak{M}}(F)$ with the induced metrics $d$ are totally geodesic and the q-dimensional distribution $\mathfrak{N}$ orthogonal to $\mathbb{F}$ is geodesically invariant.

The first statement of Theorem 1, Proposition 1 and Theorem 2 imply the following statement.

Corollary 1. Assume that a foliation $(M, F)$ satisfies the assumptions of Theorem 2 and the holonomy pseudogroup of this foliation is quasi-analytic. Then the graph $G(F)$ is identified with the graph $G_{\mathfrak{M}}(F)$ equipped with the induced metrics, the induced foliations $(G(F), \mathbb{F})$ and a foliation formed by the leaves of the canonical projections $p_{i}: G(F) \rightarrow M, i=1,2$, are totally geodesic.

Since a holonomy pseudogroup of a transversally analytic foliation $(M, F)$ is quasianalytic, the graphs $G(F)$ and $G_{\mathfrak{M}}(F)$ are identified. Since the completeness of the Riemannian manifold implies the completeness of each totally geodesic foliation on this manifold, according Proposition 4, the foliation $\mathfrak{M}$ complementary to $T F$ with respect to the orthogonality is an Ehresmann connection for $(M, F)$. This is why Theorem 1 implies the following statement.

Corollary 2. If $(M, F)$ is a transversally analytic foliation on a complete Riemmanian manifold $(M, g)$ and $G(F)$ is the graph of this foliation equipped with the induced metrics, then the induced foliation $(G(F), \mathbb{F})$ and the foliations $\left(G(F), F^{(i)}\right), i=1,2$, formed by the fibres of canonical projections $p_{i}: G(F) \rightarrow M$ are totally geodesic foliations.

Remark 1. Under the assumptions of Corollary 2, $R$. Wolak proved a totally geodesic property for the foliation formed by the fibres of only one canonical projection $p_{1}: G(F) \rightarrow M$ [10, Thm. 2].

Remark 2. The graphs of pseudo-Riemmanian foliations on pseudo-Riemmanian manifold with non-degenerate metrics on the leaves were studied by A.Yu. Dolgonosova and the author in [11.

Applying Theorem 2, we prove the following properties for the graphs of the considered class of totally geodesic foliations.

Theorem 3. Let $(M, F)$ be a foliation satisfying conditions of Theorem 2, $F^{(1)}, F^{(2)}, \mathbb{F}$ be the aforementioned foliations on the graph $G_{\mathfrak{M}}(F)$ and $L_{0}=p_{1}^{-1}(x), x \in M$, be an arbitrary fibre of the canonical projection and the graph and the leaves of the corresponding foliations are considered with the induced metrics $d$. Then

1. Each leaf $\mathbb{L}=p_{1}^{-1}(L)$ of the induced foliation $\left(G_{\mathfrak{M}}(F), \mathbb{F}\right)$ is a non-degenerately reduced pseudoRiemmanian manifold with a pair of parallel foliations $\left.F^{(1)}\right|_{\mathbb{L}}$ and $\left.F^{(2)}\right|_{\mathbb{L}}$ mutually complementary with respect to the orthogonality.

2. For each leaf $L$ in the foliation $(M, F)$ there exists a regular pseudo-Riemannian covering $f_{L}$ : $L_{0} \rightarrow L$ with the group of covering transformations isomorphic to the $\mathfrak{M}$-holonomy group $H_{\mathfrak{M}}(L)$. 
3. The group $H_{\mathfrak{M}}(L)$ acts freely diagonally and properly discontinuous on the pseudo-Riemmanian product $L_{0} \times L_{0}$ by means of an isometry group $\Psi$ and there exists an isometry

$$
\eta: \mathbb{L} \rightarrow\left(L_{0} \times L_{0}\right) / \Psi
$$

of the leaf $\mathbb{L}=p_{1}^{-1}(L)$ onto the quotient-manifold $\left(L_{0} \times L_{0}\right) / \Psi$ mapping the leaves of parallel foliations $\left.F^{(1)}\right|_{\mathbb{L}}$ and $\left.F^{(2)}\right|_{\mathbb{L}}$ onto the respected leaves of the foliations covered by the product $L_{0} \times L_{0}$.

According the second statement in Theorem 3 , each leaf $\left(L_{\alpha}, g\right)$ of the foliation $(M, F)$ is locally isometric to $\left(L_{0}, d\right)$ that yields the following corollary.

Corollary 3. If there exists a leaf $L$ of a totally geodesic foliation $(M, F)$ having a constant curvature, then each leaf $L_{\alpha}$ of this foliation has the same constant curvature.

Let $(M, g)$ be a non-degenerately reducible pseudo-Riemmanian manifold considered with the LeviCivita connection. This means that there exists a subspace $\mathfrak{M}_{x}$ in the tangential vector space $T_{x} M$ at some point $x \in M$, on which the restriction of the metrics $g$ is non-degenerate and $\mathfrak{M}_{x}$ is invariant with respect to the parallel translations along piece-wise smooth loops at the point $x$. A parallel translation of the subspace $\mathfrak{M}_{x}$ into any other point in the manifold $M$ defines a distribution $\mathfrak{M}$ on $M$ called parallel. Since the parallel translation preserves the metric tensor, then the subspace $\mathfrak{M}_{x}^{\perp}$ complementary with respect to the orthogonality is invariant with respect to parallel translations along the loops at the point $x$ and therefore, it also defines a parallel distribution $\mathfrak{M}^{\perp}$ on $M$. As it is known, each parallel distribution is integrable and is tangential to some foliation, which is called parallel.

Thus, on each non-degenerately reducible pseudo-Riemmanian manifold there exists a pair of parallel foliations $\left(F, F^{\perp}\right)$ mutually complementary with respect to the orthogonality.

Theorem 4. Let $(M, g)$ be a non-degenerately reducible pseudo-Riemannian manifold $F$ and $F^{\perp}$ be the parallel foliations of an additional dimension and $\mathfrak{M}=T F^{\perp}$ is an Ehresmann connection for the foliation $(M, F)$. Then in terms of the above notations we have:

1. The graphs $G(F)$ and $G_{\mathfrak{M}}(F)$ are canonically isomorphic and are identified; $G(F)$ is equipped with the induced pseudo-Riemmanian metrics $d$.

2. For almost all points $z \in G(F)$ and $x=p_{1}(z) \in M$ the leaves $\mathbb{L}=\mathbb{L}(z)$ and $L=L(x)$ have trivial holonomy groups and are isometric to $L_{0} \times L_{0}$ and $L_{0}$, respectively, where $L_{0}$ is an arbitrary fixed leaf of the canonical projection $p_{1}: G(F) \rightarrow M$ with the induced metrics.

3. There defined the foliations $F^{\mathfrak{N}}, \mathcal{F}^{(i)}, i=1,2$, such that $T F^{\mathfrak{N}}=\mathfrak{N}$ and $T \mathcal{F}^{(i)}=\mathfrak{M}^{(i)}$ on the graph $G(F)$.

4. The graph $G(F)$ with the induced metrics $d$ is a non-degenerately reducible pseudo-Riemmanian manifold with three pairs of parallel mutually complementary with respect to the orthogonality foliations $\left(F^{(1)}, \mathcal{F}^{(1)}\right),\left(F^{(2)}, \mathcal{F}^{(2)}\right)$, and $\left(F^{\mathfrak{N}}, \mathbb{F}\right)$.

5. Each of the above six foliations possesses an integrable Ehresmann connection and for its graph Theorems 2 and 3 hold as well as Statements 1-4 of the present theorem.

Theorem 4 and the following two statements show that the considered class of foliations is rather wide.

Proposition 2. Let $(M, F)$ be a totally geodesic foliation of a codimension $q$ on $n$-dimensional pseudo-Riemmanian manifold $(M, g)$, where $0<q<n$. If complete pseudo-Riemmanian metrics are induced on its leaves, then a q-dimensional distribution $\mathfrak{M}$ orthogonal to the leaves is an Ehresmann connection for the foliation $(M, F)$.

Proposition 3. Let $\left(B, g^{B}\right)$ be an arbitrary pseudo-Riemmanian manifold. If $(M, F)$ is a foliation obtained by the suspension of a homomorphism

$$
\rho: \pi_{1}(B, b) \rightarrow \operatorname{Diff}(T),
$$

then on $M$ there exists a pseudo-Riemmanian metrics such that

1) $(M, F)$ is a totally geodesic foliation with an induced pseudo-Riemmanian metrics on the leaves and the leaves of the foliation $(M, F)$ are complete pseudo-Riemmanian manifolds if and only if $\left(B, g^{B}\right)$ is complete; 
2) the associated locally trivial fibration is formed by the fibres of the pseudo-Riemmanian submersion $p: M \rightarrow B$;

3) the distribution formed by the tangential spaces to the fibres of the submersion $p: M \rightarrow B$ is an integrable Ehhresmann connection for $(M, F)$;

4) the graph $G(F)$ is Hausdorff if and only if the group $\Psi:=\rho\left(\pi_{1}(B, b)\right)$ acts quasi-analytically on the manifold $T$.

Notations. Following [15], we denote by $P(N, H)$ the principle $H$-bundle over a manifold $N$. By $\mathfrak{X}(M)$ we denote the module of smooth vector fields on a manifold $M$ over the algebra $\mathfrak{F}(M)$ of smooth functions. A foliation $F$ on a manifold $M$ is denoted both by a single letter and by the pair $(M, F)$. Let $\mathfrak{M}$ be a smooth distribution on the manifold $M$, then $\mathfrak{X}_{\mathfrak{M}}(M):=\left\{X \in \mathfrak{X}(M) \mid X_{u} \in \mathfrak{M}_{u} \forall u \in M\right\}$. If $\mathfrak{M}$ is integrable and $\mathfrak{M}=T F$, then $\mathfrak{X}_{\mathfrak{M}}(M)$ is also denoted by $\mathfrak{X}_{F}(M)$.

By $\mathfrak{F o l}$ we denote the category of foliations, in which the morphisms map the leaves of one foliation into those of another.

The restriction of a foliation (or metrics) on a submanifold is denoted by the symbol as the original foliation (or metrics).

$\mathrm{By} \cong$ we denote the isomorphism in a corresponding category, while $\oplus$ stands for the direct sum of vector spaces and distributions.

\section{Foliations with Ehresmann CONNECTiOn}

2.1. Ehresmann connection for foliations. Assume we are given a foliation $F$ of an arbitrary codimension $q \geqslant 1$ on a smooth $n$-dimensional manifold $M$.

We denote by $\mathfrak{M}$ a $q$-dimensional transversal to $F$ distribution, then the tangential space $T_{x} M$ to the manifold $M$ at each point $x \in M$ can be represented as $T_{x} M=T_{x} F \oplus \mathfrak{M}_{x}$. The distribution $\mathfrak{M}$ and piece-wise smooth integral curves of this distribution are called $\mathfrak{M}$-horizontal or simply horizontal. A tangent distribution $T F$ to the leaves of the foliation $F$ and each vector $X$ in $T_{x} F, x \in M$, are called vertical. A curve in the manifold $M$ belonging to a single leaf in the foliation $F$ is called vertical.

A piece-wise smooth mapping $H: I \times I \rightarrow M$, where $I=[0,1]$, is called the vertical-horizontal homotopy $(V H H)$ if for each $(s, t) \in I \times I$, the curve $\left.H\right|_{I \times\{t\}}$ is horizontal and $\left.H\right|_{\{s\} \times I}$ is a vertical curve. The pair $\left(\left.H\right|_{I \times\{0\}},\left.H\right|_{\{0\} \times I}\right)$ is called the base of VHH $H$. Two curves $(\delta, \tau)$ on $M$ are called the admissible pair of paths if $\delta(0)=\tau(0)$ and the path $\delta$ is horizontal and $\tau$ is vertical.

If for each admissible pair of paths $(\delta, \tau)$ there exists $V H H$ with the base $(\delta, \tau)$, then the distribution $\mathfrak{M}$ is called the Ehresmann connection for $F$. If at that the distribution $\mathfrak{M}$ is integrable, then the Ehresmann connection $\mathfrak{M}$ is called integrable.

We shall say that a curve $\widetilde{\delta}$ is obtained by the translation of the curve $\delta$ along $\tau$ with respect to an Ehresmann connection $\mathfrak{M}$ if $\widetilde{\delta}:=\left.H\right|_{I \times\{1\}}$. We denote this translation by $\delta \stackrel{\tau}{\rightarrow}>\widetilde{\delta}$.

2.2. $\mathfrak{M}$-holonomy groups. Let $(M, F)$ be a foliation with an Ehresmann connection $\mathfrak{M}$. We denote by $\Omega_{x}, x \in M$, the set of horizontal curves originating at the point $x$. The action of the fundamental group $\pi_{1}(L, x)$ of the leaf $L=L(x)$ on the set $\Omega_{x}$ is defined as follows: $\Phi_{x}: \pi_{1}(L, x) \times \Omega_{x} \rightarrow \Omega_{x}$ : $([h], \sigma) \mapsto \tilde{\sigma}$, where $[h] \in \pi_{1}(L, x)$ and $\tilde{\sigma}$ is the result of the translation of the curve $\sigma \in \Omega_{x}$ along $h$ with respect to $\mathfrak{M}$. Let $K_{\mathfrak{M}}(L, x)$ be the kernel of the action $\Phi_{x}$, that is, $K_{\mathfrak{M}}(L, x)=\left\{\alpha \in \pi_{1}(L, x) \mid \alpha(\sigma)=\right.$ $\left.\sigma \forall \sigma \in \Omega_{x}\right\}$. The quotient group $H_{\mathfrak{M}}(L, x)=\pi_{1}(L, x) / K_{\mathfrak{M}}(L, x)$ is called the $\mathfrak{M}$-holonomy group of the leaf $L$ [4]. Thanks to the connectivity of the leaves, the $\mathfrak{M}$-holonomy groups at different points of the same leaf are isomorphic. Let $\Gamma(L, x)$ be the germ holonomy group of a leaf $L$. There is an epimorphism of the groups $\chi: H_{\mathfrak{M}}(L, x) \rightarrow \Gamma(L, x)$ satisfying the identity

$$
\chi \circ \mu=\nu,
$$

where $\mu: \pi_{1}(L, x) \rightarrow H_{\mathfrak{M}}(L, x)$ is the quotient mapping and $\nu([h]):=<h>$ is the germ of a local holonomic diffeomorphism of a transversal $q$-dimensional disk along a loop $h$ at the point $x$.

2.3. Local horizontal holonomic diffeomorphisms. We consider an arbitrary smooth foliation $(M, F)$ of codimension $q$ on a $n$-dimensional manifold $M$. Let $\mathfrak{M}$ be a smooth $q$-dimensional distribution on $M$ transversal to this foliation, that is, $T_{x} M=T_{x} F \oplus \mathfrak{M}_{x}$ for all $x \in M$. In what follows we shall consider vertical-horizonatl homotopies with respect to the distributions $T M$ and $\mathfrak{M}$. At each point $x \in M$ there exists a neighbourhood $V_{x}$ such that for each admissible pair of paths $(\sigma, h)$ in 
$V_{x}$ with a common origin at $x$, there exists a $V H H$ in $V_{x}$ with the base $(\sigma, h)$. Let $\sigma:[0,1] \rightarrow M$ be arbitrary smooth integral curve of the distribution $\mathfrak{M}$ with the origin $x_{0}:=\sigma(0)$ and the end $x_{1}:=\sigma(1)$. It is easy to make sure that there exist contractible neighbourhoods $U_{0}$ of the point $x_{0}$ in the leaf $L_{0} \ni x_{0}$ and $U_{1}$ of the point $x_{1}$ in the leaf $L_{1} \ni x_{1}$ such that for each point $x \in U_{0}$ and each piece-wise smooth path $h_{x}:[0,1] \rightarrow U_{0}$ connecting $h(0)=x_{0}$ with $h(1)=x$, there exists $V H H$ $H_{x}$ with the base $\left(\sigma, h_{x}\right)$. At that, $\mathfrak{M}$-horizontal curve $\sigma_{x}(s):=H_{x}(s, 1), s \in[0,1]$, is smooth and since $U_{0}$ is contractible, this curve is independent of the choice of the path $h_{x}$ connecting $x_{0}$ with $x$ in $U_{0}$. In what follows $\sigma_{x}$ is called the translation of the path $\sigma$ to the point $x \in U_{0}$. At that, the diffeomorphism is well-defined:

$$
\Phi_{\sigma}: U_{0} \rightarrow U_{1}: x \mapsto \sigma_{x}(1), \quad x \in U_{0},
$$

which is called the locally horizontal holonomic diffeomorphism along $\mathfrak{M}$-curve $\sigma$ [5].

The definition of the Lie derivative $L_{X} g$ of 2 -form $g$ along a vector field $X$ implies the following statement.

Lemma 1. Assume that $(M, F)$ is a foliation of codimension $q$ on $n$-dimensional pseudoRiemmanian manifold $(M, g)$ and the induced metrics does not degenerate on its leaves, and that $\mathfrak{M}$ is the q-dimensional distribution orthogonal to TF. Let $\sigma:[0,1] \rightarrow M$ be an arbitrary smooth $\mathfrak{M}$-horizontal curve in $M, \Phi_{\sigma}: U_{0} \rightarrow U_{1}$ be a local holonomic diffeomorphism along $\sigma$ and $W:=\left\{\sigma_{x}(s) \mid x \in U_{0}, s \in(0,1)\right\}$, where $\sigma_{x}$ is the translation of $\sigma$ to the point $x \in U_{0}$. Then the following two conditions are equivalent:

1. The diffeomorphism $\Phi_{\sigma}$ is an isometry of $\left(U_{0}, g\right)$ and $\left(U_{1}, g\right)$;

2. For a vector field $X \in \mathfrak{X}_{\mathfrak{M}}(W)$ such that $\left.X\right|_{\sigma_{x}(s)}=\dot{\sigma}_{x}(s)$, where $x \in U_{0}, s \in[0,1]$, the identity $\left(L_{X} g\right)(Y, Z)=0$ holds for all $Y, Z \in \mathfrak{X}_{F}(W)$.

\section{Pseudo-Riemmanian submersions}

The study of pseudo-Riemmanian submersions was initiated by B. O'Neill [12] and A. Gray [13]. A smooth surjective submersion $p: M \rightarrow B$ between two pseudo-Riemannian manifolds $(M, g)$ and $\left(B, g^{B}\right)$ is called the pseudo-Riemmanian submersion if the metrics induced on each fibre of the submersion $p^{-1}(b)$, where $b \in B$ is non-degenerate and $p$ preserves the scalar product of the vectors orthogonal to the fibres of the submersion.

Numerous works by various authors are devoted to studying pseudo-Riemmanian submersions. Pseudo-Riemannian submersions with totally geodesic fibres are of a special interest, for some classes classification theorems were proved, see [14] and the references therein.

The following properties of pseudo-Riemannian submersions with totally geodesic fibres are essentially employed in the present work.

Proposition 4. If $(M, g)$ and $\left(B, g^{B}\right)$ are pseudo-Riemannian manifolds of dimensions $n$ and $q$, respectively, where $0<q<n$, and $p: M \rightarrow B$ is a pseudo-Riemannian submersion with fibres being totally geodesic manifolds in $(M, g)$, then

(i) the projection $\sigma=p \circ \gamma$ of each geodesic curves $\gamma$ in $(M, g)$ is geodesic in $\left(B, g^{B}\right)$;

(ii) the pre-image $p^{-1}(L)$ of each totally geodesic submanifold $L$ from the base $\left(B, g^{B}\right)$ is a totally geodesic submanifold in $(M, g)$; it is not connected once the lfibres of the submersion $p$ are not.

Proof. Let $p: M \rightarrow B$ be a pseudo-Riemannian submersion with totally geodesic fibres.

(i). The geodesic property of a curve in a pseudo-Riemannian manifold $(M, g)$ is local. This is why it is sufficient to show that for each geodesic curve $\gamma$ in an arbitrary coordinate neighbourhood $U$ adapted to $(M, F)$, its projection $p \circ \gamma$ is a geodesic curve in the neighbourhood $V:=p(U)$ of the manifold $\left(B, g^{B}\right)$. We observe that $\left.p\right|_{U}: U \rightarrow V$ is a pseudo-Riemannian submersion on a contractible manifold with contractible fibres. This is why without loss of generality, in the present proof we let $M=U, B=V, p: M \rightarrow B$ be a pseudo-Riemannina submersion with totally geodesic fibres, and $F=\left\{p^{-1}(b) \mid b \in B\right\}$.

Assume that pseudo-Riemannian metrics $g$ and $g^{B}$ have the same signatures $(k, s)$ and $\left(k_{1}, s_{1}\right)$, respectively, where $k+s=n, k_{1}+s_{1}=q$. Let $H_{1}=O\left(k_{1}, s_{1}\right), H=O(k, s)$. By $\mathcal{L}(M, H)=M \times H$ and $P_{1}\left(B, H_{1}\right)=B \times H_{1}$ we denote the principle bundles of pseudo-orthogonal frames on $M$ and $B$ defined by the metrics $g$ and $g^{B}$, respectively; they are trivial principal bundles with projections 
$\pi: \mathcal{L} \rightarrow M$ and $f_{1}: P_{1} \rightarrow B$. Let $\mathfrak{M}$ be a $q$-dimensional distribution orthogonal to the fibres of the submersion $p$. We denote by $P=M \times H_{1}$ the bundle of $\mathfrak{M}$-transversal frames being the pre-image of the bundle $P_{1}\left(B, H_{1}\right)$ under the submersion $p$, that is, $P=\left\{(y, v) \in M \times P_{1} \mid p(y)=f_{1}(v)\right\}$. At that, the projections $f: P \rightarrow M, f(y, v):=y, h: P \rightarrow P_{1}, h(y, v)=v$ for all $(y, v) \in P$, are well-defined and they satisfy the identity $f_{1} \circ h=p \circ f$. The fibres of the submersion $h: P \rightarrow P_{1}$ form the foliation $\left(P, F_{P}\right)$.

Since $M=U$ is a coordinate neighbourhood adapted to the foliation $(M, F)$, at each point $y \in M$, a coordinate frame $\left(\left.\frac{\partial}{\partial x^{a}}\right|_{y},\left.\frac{\partial}{\partial x^{\alpha}}\right|_{y}\right), a=1, \ldots, n-q, \alpha=n-q+1, \ldots, n$, is well-defined and $\left\{\left.\frac{\partial}{\partial x^{a}}\right|_{y}\right\}$ is a basis of $T_{y} F$ in the tangent space to the leaf of the foliation $(M, F)$ at the point $y$. Each point $(y, v) \in P$ is a transversal frame, that is, a basis $\left\{\left.Z_{\alpha}\right|_{y}\right\}$ in the vector space $\mathfrak{M}_{y}$ at the point $y \in M$ defined by the identity $p_{* y}\left(\left.Z_{\alpha}\right|_{y}\right)=\left.X_{\alpha}\right|_{x}$, where $\left\{\left.X_{\alpha}\right|_{x}\right\}=v$ is the basis in the tangential vector space $T_{x} B$ at the point $x=f_{1}(v)=p(y)$. Therefore, the mapping $J: P \rightarrow \mathcal{L}, J(y, v)=\left\{\left.\frac{\partial}{\partial x^{a}}\right|_{y},\left.Z_{\alpha}\right|_{y}\right\}$ is well-defined and this is an embedding of the manifold $P$ into $\mathcal{L}$ satisfying the identity $\pi \circ J=f$.

Let $E_{n-q}$ be the unit $(n-q)$-dimensional matrix. By $j: H_{1} \rightarrow H: A \mapsto\left(\begin{array}{cc}E_{n-q} & 0 \\ 0 & A\end{array}\right)$ we denote an embedding of the group $H_{1}$ into the group $H$. At that, the pair $(J, j)$ defines a reduction $\mathcal{R}$ of $H$ foliation $\mathcal{L}$ to a closed subgroup $j\left(H_{1}\right)$ [15, Ch. 1, Sect. 5]. Since the mapping $J: P \rightarrow \mathcal{R}=J(P)$ is an isomorphism of principle foliated spaces can be identified with $P$ with $\mathcal{R}$ by means with $J$. At that, we have a commutative diagram:

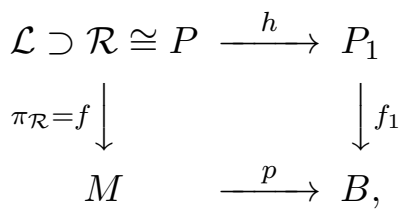

where $\pi_{\mathcal{R}}:=\left.\pi\right|_{\mathcal{R}}$. Hence, on $\mathcal{R}$, the foliation $\mathcal{F}$ is well-defined as the image of the foliation $\left(P, F_{P}\right)$ under the mentioned identification and the restriction of the mapping $\pi_{\mathcal{R}}$ on each leaf $(\mathcal{R}, \mathcal{F})$ is a diffeomorphism on a corresponding leaf $(M, F)$.

The Levi-Civita connection $\nabla$ of the pseudo-Riemannian manifold $(M, g)$ defines a $H$-connection $Q$ on $\mathcal{L}$. Let $Q^{(1)}$ be a $H_{1}$-connection on $P_{1}$ defined by the Levi-Civita connection $\nabla^{B}$ of the pseudoRiemannian manifold $\left(B, g^{B}\right)$. We denote by $\omega$ the $\mathfrak{g l}(n, \mathbb{R})$-valued 1 -form of the connection $Q$, while $\theta$ stands for the canonical 1-form of this connection on $\mathcal{L}$ with values in $\mathbb{R}^{n}$. We recall that $B_{\xi} \in \mathfrak{X}(\mathcal{L})$ is called the standard horizontal vector field if $\omega\left(B_{\xi}\right)=0$, that is, $B_{\xi} \in \mathfrak{X}_{Q}(\mathcal{L})$, and $\theta\left(B_{\xi}\right)=\xi=$ const $\in \mathbb{R}^{n}$. As it is known [15, Ch. III, Prop. 6.3], a curve $\gamma$ is geodesic in $(M, \nabla)$ if and only if $\gamma$ is a projection of an integral curve of some standard horizontal vector field.

Since the lift in $\mathcal{L}$ of each geodesic curve in $(M, \nabla)$ is an integral curve of the distribution $Q$, the complete geodesicity of $(M, F)$ yields the inclusion $\left.T \mathcal{F} \subset Q\right|_{\mathcal{R}}$. Therefore, $\left.Q\right|_{\mathcal{R}}=T \mathcal{F} \oplus \mathfrak{N}$, where $\mathfrak{N}=\left.\pi^{*} \mathfrak{M} \cap Q\right|_{\mathcal{R}}$

Since $p: M \rightarrow B$ is a pseudo-Riemannian submersion, according [11, Thm. 1], the distribution $\mathfrak{M}$ is geodesically invariant and this is why the $Q$-lift $\widetilde{\gamma}$ to a point $u \in \pi^{-1}\left(\gamma_{0}\right) \cap \mathcal{R}$ of each $\mathfrak{M}$-horizontal geodesic curve $\gamma:[0,1] \rightarrow M$ is an integral curve of the distribution $\mathfrak{N}$. Moreover, for each vector $Y \in \mathfrak{N}_{u}, u \in \mathcal{R}$ such that $\theta(Y)=\xi \in\left\{0_{n-q}\right\} \times \mathbb{R}^{q}$, where $0_{n-q}$ is the zero in $\mathbb{R}^{n-q}$, there exists a unique $\mathfrak{M}$-horizontal geodesic curve $\gamma$ on $M$, whose $Q$-lift at the point $u$ is an integral curve of a standard horizontal vector field $B_{\xi}$ such that $\left.B_{\xi}\right|_{u}=Y$.

By properties of a pseudo-Riemannian submersion, each $\mathfrak{M}$-horizontal geodesic curve is projected to a geodesic curve of the base $\left(B, \nabla^{B}\right)$ by means of $p: M \rightarrow B$ [12]. Hence, in view of the identity $f_{1} \circ h=p \circ \pi_{\mathcal{R}}$, we obtain $h_{* u}\left(\mathfrak{N}_{u}\right)=h_{* u}\left(Q_{u}\right)=Q_{h(u)}^{(1)}$ for each point $u \in \mathcal{R}$.

Let $\gamma$ be an arbitrary geodesic curve in $(M, g)$ passing through a point $x=\gamma(0)$ along a vector $X=\dot{\gamma}(0) \in T_{x} M$. We take a point $u_{0} \in \mathcal{R}$ such that $\pi\left(u_{0}\right)=x$. We treat the frame $u_{0}$ as a mapping $u_{0}: \mathbb{R}^{n} \rightarrow T_{x} M$, which maps a vector in $\mathbb{R}^{n}$ with coordinates $\lambda_{1}, \ldots, \lambda_{n}$ in the standard basis in $\mathbb{R}^{n}$ into the vector in $T_{x} M$ with the same coordinates in the base $u_{0}$. Assume that $\eta:=u_{0}^{-1}(X) \in \mathbb{R}^{n}$. Let $p r: \mathbb{R}^{n} \cong \mathbb{R}^{n-q} \times \mathbb{R}^{q} \rightarrow \mathbb{R}^{q}$ be the canonical projection onto the factor and $\xi:=\operatorname{pr}(\eta) \in \mathbb{R}^{q}$. Since $\gamma$ is a geodesic curve, there exists an integral curve $\widehat{\gamma}$ of the standard vector field $B_{\eta}$ on $\mathcal{R}$ with the origin at the point $\widehat{\gamma}(0)=u_{0}$. At that $\gamma=\pi \circ \widehat{\gamma}$ and $\widehat{\sigma}:=h \circ \widehat{\gamma}$ is an integral curve of the standard vector field $B_{\xi}$ on $P_{1}$ passing through the point $v_{0}=h\left(u_{0}\right)=\widehat{\sigma}(0)$. Since $p \circ \pi_{\mathcal{R}}=f_{1} \circ h$, we have the chain of the identities $\sigma=p \circ \gamma=p \circ(\pi \circ \widehat{\gamma})=(p \circ \pi) \circ \widehat{\gamma}=\left(f_{1} \circ h\right) \circ \widehat{\gamma}=f_{1} \circ(h \circ \widehat{\gamma})=f_{1} \circ \widehat{\sigma}$. 
Thus, the curve $\sigma:=f_{1} \circ \widehat{\sigma}$ is geodesic on $\left(B, g^{B}\right)$ and it is a projection of the geodesic curve $\gamma$ of the manifold $(M, g)$, that is, $p \circ \gamma=\sigma$, and this proves Statement (i).

(ii). Let $L$ be a totally geodesic submanifold in $\left(B, g^{B}\right)$ and $N:=p^{-1}(L)$ be a smooth embedded submanifold in $M$; it is not connected once the fibres of the submersion $p: M \rightarrow B$ are not. We take an arbitrary point $y \in N$ and a vector $Y \in T_{y} N$. Let $\gamma=\gamma_{Y}(s), s \in[0,1]$, be a geodesic curve in $(M, g)$ passing through the point $y=\gamma_{Y}(0)$ along the vector $Y$, that is, $Y=\dot{\gamma}_{Y}(0)$. According the proven statement $(i)$, the curve $\sigma:=p \circ \gamma$ is geodesic $\left(B, g^{B}\right)$ and it passes through the point $b=p(y)=\sigma(0)$ along the vector $X=p_{* y} Y \in T_{b} L$. Since $L$ is a totally geodesic submanifold, then $\sigma(s) \in L$ for all $s \in[0,1]$, and hence, $\gamma(s) \in N$ for all $s \in[0,1]$. This means that the submanifold $N$ in $(M, g)$ is totally geodesic. The proof is complete.

\section{Proof of Theorem 2}

\subsection{Criterion of a total geodesicity of foliation.}

Definition 4. A vector field $X$ on a manifold $M$ is called foliated with respect to a foliation $(M, F)$ if $[X, Y] \in \mathfrak{X}_{F}(M)$ for each $Y \in \mathfrak{X}_{F}(M)$.

Proposition 5. Let $(M, F)$ be a foliation on a pseudo-Riemannian manifold $(M, g)$ with the LeviCivita connection $\nabla$ and on the leaves of the foliation, pseudo-Riemannian metrics are induced, and let $\mathfrak{M}$ be the distribution complementary to TF with respect to the orthogonality. Then the following statements are equivalent:

(1) the foliation $(M, F)$ is totally geodesic;

(2) $L_{X} g(Y, Z)=0$ for arbitrary vector fields $X \in \mathfrak{X}_{\mathfrak{M}}(M)$ and $Y, Z \in \mathfrak{X}_{F}(M)$;

(3) $L_{X} g(Y, Z)=0$ for each foliated vector field $X \in \mathfrak{X}_{\mathfrak{M}}(M)$ and for each vector fields $Y, Z \in$ $\mathfrak{X}_{F}(M)$;

(4) for each $\mathfrak{M}$-horizontal curve $\sigma:[0,1] \rightarrow M$, the local horizontal holonomic diffeomorphism $\Phi_{\sigma}: U_{0} \rightarrow U_{1}$ is an isometry between $\left(U_{0}, g\right)$ and $\left(U_{1}, g\right)$.

Proof. We denote by $\alpha_{\mathfrak{M}}(Y, Z)$ the orthogonal projection of $\nabla_{Y} Z$ into $\mathfrak{X}_{\mathfrak{M}}(M)$ with respect to the decomposition $T M=T F \oplus \mathfrak{M}$. The total geodesicity of the foliation $(M, F)$ is equivalent to $\alpha_{\mathfrak{M}}(Y, Z)=$ 0 for all $Y, Z \in \mathfrak{X}_{F}(M)$.

The equivalence of (1) and (2) was proved in [16, Prop. 2.7] as follows. By employing the property of the Levi-Civita connection $\nabla$ of the pseudo-Riemannian manifold $(M, g)$, the identity was obtained:

$$
L_{X} g(Y, Z)=g\left(X, \alpha_{\mathfrak{M}}(Y, Z)\right) \quad \text { for all } \quad X \in \mathfrak{X}_{\mathfrak{M}}(M), \quad Y, Z \in \mathfrak{X}_{F}(M) .
$$

And since the induced metrics on the leaves is non-degenerate, this implies the equivalence

$$
L_{X} g(Y, Z)=0 \quad \text { for all } \quad X \in \mathfrak{X}_{\mathfrak{M}}(M) \quad \Leftrightarrow \quad \alpha_{\mathfrak{M}}(Y, Z)=0,
$$

that is, $(1) \Leftrightarrow(2)$.

The implication $(2) \Rightarrow(3)$ is obvious. Assume that (3) holds. We observe that an arbitrary vector field $X \in \mathfrak{X}_{\mathfrak{M}}(M)$ is a linear combination of foliated vector fields in $\mathfrak{X}_{\mathfrak{M}}(M)$, that is, $X=\beta^{k} X_{k}$, where $X_{k} \in \mathfrak{X}_{\mathfrak{M}}(M)$ are foliated vector fields, $\beta^{k} \in \mathfrak{F}(M)$. Applying (5), thanks to the bilinearity of $g$, we obtain

$$
L_{X} g(Y, Z)=g\left(X, \alpha_{\mathfrak{M}}(Y, Z)\right)=g\left(\beta^{k} X_{k}, \alpha_{\mathfrak{M}}(Y, Z)\right)=\beta^{k} g\left(X_{k}, \alpha_{\mathfrak{M}}(Y, Z)\right)=\beta^{k} L_{X_{k}} g(Y, Z) .
$$

According the assumption, $L_{X_{k}} g(Y, Z)=0$ and this is why $L_{X} g(Y, Z)=0$. Therefore, $(3) \Rightarrow(2)$ and $(2) \Leftrightarrow(3)$.

If $\sigma$ is a piece-wise smooth $\mathfrak{M}$-horizontal curve, then $\Phi_{\sigma}$ is a composition of local horizontal holonomic diffeomorphisms corresponding to smooth pieces of the curve $\sigma$. This is why, without loss of generality, in (4) we can assume that $\sigma:[0,1] \rightarrow M$ is a smooth $\mathfrak{M}$-horizontal curve.

Assume that (3) is satisfied. Let $\sigma:[0,1] \rightarrow M$ be a smooth $\mathfrak{M}$-horizontal curve, $\Phi_{\sigma}: U_{0} \rightarrow U_{1}$ be a local horizontal holonomic diffeomorphism along $\sigma$ and $X$ be a vector field induced by $\Phi_{\sigma}$ in the way described in Lemma 1. Since $X$ is a foliated vector field orthogonal to the foliation $(M, F)$, it follows from (3) that $L_{X} g(Y, Z)=0$ for all $Y, Z \in \mathfrak{X}_{F}(M)$. Therefore, by Lemma 1, $\Phi_{\sigma}$ is an isometry between $\left(U_{0}, g\right)$ and $\left(U_{1}, g\right)$. Thus, $(3) \Rightarrow(4)$.

Now it is sufficient to show that $(4) \Rightarrow(3)$. Let $X \in \mathfrak{X}_{\mathfrak{M}}(M)$ be an arbitrary foliated vector field and $\sigma:[0,1] \rightarrow M$ be its arbitrary integral curve. Since $\sigma$ is a $\mathfrak{M}$-horizontal curve, then a local horizontal 
holonomic diffeomorphism $\Phi_{\sigma}: U_{0} \rightarrow U_{1}$ along $\sigma$ is well-defined. Since $X$ is a foliated vector field, the translation $\sigma_{x}$ of the curve $\sigma$ at the point $x \in U_{0}$ is also an integral curve of the field $X$. According Lemma 1, this implies that $L_{X} g(Y, Z)=0$ for all $Y, Z \in \mathfrak{X}_{F}(M)$. Thus, (4) $\Rightarrow(3)$.

Remark 3. Proposition 5 has been proved without assuming the existence of the Ehresmann connection for the foliation $(M, F)$.

4.2. Total geodesicity of foliation $F^{(1)}$. Let us prove that $F^{(1)}$ is a totally geodesic foliation on the graph $\left(G_{\mathfrak{M}}(F), d\right)$.

Let $\gamma:[0,1] \rightarrow G_{\mathfrak{M}}(F)$ be a smooth $\mathfrak{N}$-curve in $G_{\mathfrak{M}}(F)$ with the origin at the point $\gamma(0)=z_{0}=\left(x_{0},\{h\}, y_{0}\right) \in G_{\mathfrak{M}}(F)$. Then $\sigma:=p_{1} \circ \gamma$ is a $\mathfrak{M}$-curve in $M$ with the origin at the point $x_{0}=\sigma(0)=p_{1}\left(z_{0}\right)$. Since $(\sigma, h)$ is an admissible pair, there exists a VHH $H$ with a base $(\sigma, h)$. Let $\sigma \stackrel{h}{\rightarrow}>\tilde{\sigma}$, at that $\tilde{\sigma}=p_{2} \circ \gamma$. Without loss of generality we assume that $V_{0}$ is a neighbourhood of the point $z_{0}$ in the fibre $p_{1}^{-1}\left(x_{0}\right)$ such that the neighbourhood $U_{0}=p_{2}\left(V_{0}\right)$ belonging to the leaf $L=L\left(x_{0}\right)$ is properly covered by the mapping $\left.p_{2}\right|_{L^{(1)}}: L^{(1)} \rightarrow L$, where $L^{(1)}=L^{(1)}\left(z_{0}\right)=p_{1}^{-1}\left(x_{0}\right)$. If $\Phi_{\gamma}: V_{0} \rightarrow V_{1}: z \mapsto \Phi_{\gamma}(z)$ is a horizontal holonomic diffeomorphism along $\gamma$ in $G_{\mathfrak{M}}(F)$, where $V_{1}$ is a neighbourhood of the point $\gamma(1)$ in the leaf $p_{1}^{-1}(\sigma(1))$, then for each point $z \in V_{0}$ and the $\mathfrak{N}$-lift $\gamma_{z}$ of the curve $\sigma$ at the point $z$, by the definition we have $\Phi_{\gamma}(z)=\gamma_{z}(1)$. At that, $z=\left(x_{0},\left\{h_{y}\right\}, y\right)$, where $y=p_{2}(z), h_{y}=h \cdot t_{y}, t_{y}$ is a path in $U_{0}$ connecting $y_{0}$ with $y$ in $U_{0}$. We observe that $\sigma_{y}=p_{2} \circ \gamma_{z}$ is $\mathfrak{M}$ curve in $M$ obtained by translating $\sigma$ along the path $h_{y}$ and $\Phi_{\tilde{\sigma}}:=p_{2} \circ \Phi_{\gamma}: U_{0}=p_{2}\left(V_{0}\right) \rightarrow U_{1}=p_{2}\left(V_{1}\right)$ is a local $\mathfrak{M}$-horizontal holonomic diffeomorphism along $\tilde{\sigma}$ satisfying a commutative diagram:

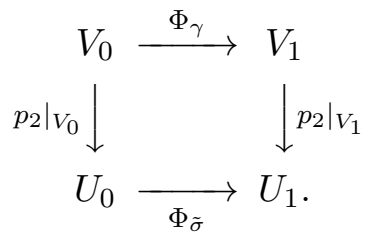

According Proposition 5 , the total geodesicity of the foliation $F$ implies that $\Phi_{\tilde{\sigma}}: U_{0} \rightarrow U_{1}$ is an isometry between the pseudo-Riemannian manifolds $\left(U_{0}, g\right)$ and $\left(U_{1}, g\right)$. Hence, taking into consideration that $\left.p_{1}\right|_{V_{0}}: V_{0} \rightarrow U_{0},\left.p_{2}\right|_{V_{1}}: V_{1} \rightarrow U_{1}$ are isometries, by the commutativity of diagram (6) we obtain that $\Phi_{\gamma}: V_{0} \rightarrow V_{1}$ is an isometry between $\left(V_{1}, d\right)$ and $\left(V_{2}, d\right)$. Hence, similar to Proposition 5, we obtain

$$
\left(L_{X} d\right)(Y, Z)=0 \quad \text { for all } \quad X \in \mathfrak{X}_{\mathfrak{N}}\left(G_{\mathfrak{M}}(F)\right), \quad Y, Z \in \mathfrak{X}_{F^{(1)}}\left(G_{\mathfrak{M}}(F)\right) .
$$

We are going to show that $\left(L_{X} d\right)(Y, Z)=0$ for all $Y, Z \in \mathfrak{X}_{F^{(1)}}\left(G_{\mathfrak{M}}(F)\right)$ and for each $X \in$ $\mathfrak{X}_{F^{(2)}}\left(G_{\mathfrak{M}}(F)\right)$. We denote by $L^{(i)}=L^{(i)}\left(z_{0}\right), i=1,2$, and $\mathbb{L}=\mathbb{L}\left(z_{0}\right)$ the leaves in the foliations $F^{(i)}$ and $\mathbb{F}$ respectively passing through $z_{0}$. Let $\gamma$ be an arbitrary $T F^{(2)}$-curve with the origin at $z_{0}=\gamma(0)$, that is, $\gamma(s) \in L^{(2)}, s \in[0,1]$. A local horizontal diffeomorphism $\Phi_{\gamma}: W_{0} \rightarrow W_{1}$ along $\gamma$ is well-defined, where $W_{0}$ is a neighbourhood of the point $z_{0}$ in the leaf $L^{(1)}$, and $W_{1}$ is a neighbourhood of the point $z_{1}=\gamma(1)$ in the leaf $L^{(1)}\left(z_{1}\right)$ in the foliation $F^{(1)}$ passing through $z_{1}$.

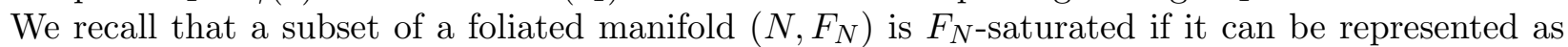
the union of some leaves in the foliation $\left(N, F_{N}\right)$.

Each leaf $\mathbb{L}$ of the induced foliation $\left(G_{\mathfrak{M}}(F), \mathbb{F}\right)$ is both $F^{(1)}$-saturated and $F^{(2)}$-saturated submanifold of the graph $G_{\mathfrak{M}}(F)$ and according the definition of the metrics $d$, the leaf $\mathbb{L}$ with the induced pseudo-Riemannian metrics $(\mathbb{L}, d)$ is locally a pseudo-Riemannian product of pseudo-Riemanninan manifolds $\left(L^{(1)}, d\right)$ and $\left(L^{(2)}, d\right)$. This implies that $\left.F^{(1)}\right|_{\mathbb{L}}$ and $\left.F^{(2)}\right|_{\mathbb{L}}$ are parallel foliations on an nondegenerate reducible pseudo-Riemannina manifold $(\mathbb{L}, d)$, see, for instance, 19 . Therefore, $\left.F^{(1)}\right|_{\mathbb{L}}$ and $\left.F^{(2)}\right|_{\mathbb{L}}$ are totally geodesic foliations on $(\mathbb{L}, d)$ and according Proposition $5, \Phi_{\gamma}: W_{0} \rightarrow W_{1}$ is an isometry and the identity holds

$$
\left(L_{X} d\right)(Y, Z)=0 \quad \text { for all } \quad X \in \mathfrak{X}_{F^{(2)}}\left(G_{\mathfrak{M}}(F)\right), \quad Y, Z \in \mathfrak{X}_{F^{(1)}}\left(G_{\mathfrak{M}}(F)\right) .
$$

We observe that at each point of the graph $G_{\mathfrak{M}}(F)$ there exists a neighbourhood $\mathcal{W}$ adapted to the foliations $\mathbb{F}, F^{(1)}$ and $F^{(2)}$ simultaneously, at which both identities (7) and (8) hold. Since $\mathfrak{M}^{(1)}=\mathfrak{N} \oplus T F^{(2)}$, each vector field $X \in \mathfrak{X}_{\mathfrak{M}^{(1)}}(W)$ in the neighbourhood $\mathcal{W}$ of an arbitrary point 
$z \in G_{\mathfrak{M}}(F)$ can be represented as $X=\alpha X^{\mathfrak{N}}+\beta X^{(2)}$, where $X^{\mathfrak{N}} \in \mathfrak{X}_{\mathfrak{N}}\left(G_{\mathfrak{M}}(F)\right), X^{(2)} \in \mathfrak{X}_{F^{(2)}}\left(G_{\mathfrak{M}}(F)\right)$, $\alpha, \beta \in \mathfrak{F}(W)$. As in the proof of Proposition 5, we show that

$$
\left(L_{\alpha X^{\mathfrak{N}}+\beta X^{(2)}} d\right)(Y, Z)=\alpha\left(L_{X^{\mathfrak{N}}} d\right)(Y, Z)+\beta\left(L_{X^{(2)}} d\right)(Y, Z) \quad \text { for all } \quad Y, Z \in \mathfrak{X}_{F^{(1)}}\left(G_{\mathfrak{M}}(F)\right) .
$$

Identities (7) and (8) imply that $\left(L_{X^{\mathfrak{r}}} d\right)(Y, Z)=0$ and $\left(L_{X^{(2)}} d\right)(Y, Z)=0$. Thus, we obtain:

$$
\left(L_{X} d\right)(Y, Z)=0 \quad \text { for all } \quad X \in \mathfrak{X}_{\mathfrak{M}^{(1)}}\left(G_{\mathfrak{M}}(F)\right), \quad Y, Z \in \mathfrak{X}_{F^{(1)}}\left(G_{\mathfrak{M}}(F)\right) .
$$

According Proposition 5 , this means that $F^{(1)}$ is a totally geodesic foliation.

4.3. Geodesic invariance of distributions $\mathfrak{M}^{(1)}, T \mathbb{F}$ and $T F^{(2)}$. The definition of the metrics $d$ implies that $p_{1}: G_{\mathfrak{M}} \rightarrow M$ is a pseudo-Riemannian submersion and $\mathfrak{M}^{(1)}$ is a distribution complementary to the leaves of this submersion with respect to the orthogonality. This is why according [11, Thm. 1], the distribution $\mathfrak{M}^{(1)}$ is geodesically invariant.

As it has been proved above, the foliation $F^{(1)}$ is totally geodesic and hence, $p_{1}: G_{\mathfrak{M}}(F) \rightarrow M$ is a pseudo-Riemannian submerions with totally geodesic fibres. Applying Statement (ii) of Proposition 4 , we obtain that the total geodesicity of $(M, F)$ implies the same for the induced foliation $\left(G_{\mathfrak{M}}(F), \mathbb{F}\right)$, since each its leaf $\mathbb{L}=p_{1}^{-1}(L)$ is the pre-image of some leaf $L$ of the foliation $(M, F)$ being a totally geodesic submanifold in $(M, g)$.

Since $T F^{(2)}=\mathfrak{M}^{(1)} \cap T \mathbb{F}$, then $T F^{(2)}$ is a geodesically invariant distribution as the intersection of geodesically invariant distributions $\mathfrak{M}^{(1)}$ and $T \mathbb{F}$. Therefore, $\left(G_{\mathfrak{M}}(F), F^{(2)}\right)$ is a totally geodesic foliation on $\left(G_{\mathfrak{M}}(F), d\right)$.

\section{Proof of Theorem 3}

1. The first statement of Theorem 3 was proved in the proof of Theorem 2, see Subsection 4.2).

2. Let $x_{1}$ and $x_{2}$ be two points on the pseudo-Riemannina manifold $(M, g)$. According $[4, \mathrm{Lm}$. 1.1], the leaves $L_{1} \ni x_{1}$ and $L_{2} \ni x_{2}$ can be connected by a $\mathfrak{M}$-horizontal curve $\sigma:[0,1] \rightarrow M$, where $y_{1}=\sigma(0) \in L_{1}, y_{2}=\sigma(1) \in L_{2}$. We connect $x_{i}$ with $y_{i}$ by the curve $\sigma_{i}$ in the leaf $L_{i}, i=1,2$. Then the product of the paths $\gamma:=\sigma_{1} \cdot \sigma \cdot \sigma_{2}^{-1}$ connects a point $x_{1}$ with a point $x_{2}$. As it is known [7, Lm. 1], each $\mathfrak{M}$-horizontal curve in $M$ possesess $\mathfrak{N}$-horizontal lifts in $G_{\mathfrak{M}}(F)$. The curves $\sigma_{1}$ and $\sigma_{2}$ possess $T F^{(2)}$-horizontal lifts in $G_{\mathfrak{M}}(F)$, since for each leaf $\mathbb{L}$ in the induced foliation $\left(G_{\mathfrak{M}}(F), \mathbb{F}\right)$, the distribution $\left.T F^{(2)}\right|_{\mathbb{L}}$ is an Ehresmann connection for the submersion $\left.p_{1}\right|_{\mathbb{L}}$. Therefore, for each point $z$ in $p_{1}^{-1}\left(x_{1}\right)$, there exists a $\mathfrak{M}^{(1)}$-horizontal lift $\widehat{\gamma}$ of the curve $\gamma$ with the origin at $\widehat{\gamma}(0)=z$ and the end $\widehat{\gamma}(1) \in p_{1}^{-1}\left(x_{1}\right)$. Since

$$
\Phi_{\widehat{\gamma}}: p_{1}^{-1}\left(x_{0}\right) \rightarrow p_{1}^{-1}\left(x_{1}\right): z \mapsto \widehat{\gamma}(1)
$$

is a horizontal holonomic diffeomorphism with respect to the foliation $\left(G_{\mathfrak{M}}, F^{(1)}\right)$ along a $\mathfrak{M}^{(1)}$ horizontal path $\widehat{\gamma}$, then by the complete geodesicity of the mentioned foliation, it follows from Proposition 5 that the mapping $\Phi_{\widehat{\gamma}}$ is an isometry. Therefore, there exists a pseudo-Riemannian manifold $L_{0}^{(1)}$ isometric to each fibre of the submersion $p_{1}$. In the same way, there exists a pseudo-Riemannian manifold $L_{0}^{(2)}$ isometric to each fibre in the submersion $p_{2}$. We fix a point $z_{0}=\left(x_{0},\left\{1_{x_{0}}\right\}, x_{0}\right) \in G_{\mathfrak{M}}(F)$, where $1_{x_{0}}$ is the constant path at the point $x_{0}$. We note that the restriction of the inversion $i: G_{\mathfrak{M}}(F) \rightarrow G_{\mathfrak{M}}(F), i(x,\{h\}, y)=\left(y,\left\{h^{-1}\right\}, x\right)$ on the leaf $p_{1}^{-1}\left(x_{0}\right)$ is an isometry $p_{1}^{-1}\left(x_{0}\right)$ onto the leaf $p_{2}^{-1}\left(x_{0}\right)$. This implies that $L_{0}^{(1)}$ and $L_{0}^{(2)}$ are isometric. We denote by $L_{0}$ a pseudo-Riemannian manifold isometric to $L_{0}^{(i)}, i=1,2$.

Let $L=L(x), x \in M$, be an arbitrary leaf in the foliation $(M, F)$. The definition of the graph $G_{\mathfrak{M}}(F)$ implies that the restriction of the canonical projection $\left.p_{1}\right|_{p_{2}^{-1}(x)}: p_{2}^{-1}(x) \rightarrow L$ is a regular covering mapping with a group of covering mappings isomorphic to the $\mathfrak{M}$-holonomy group $H_{\mathfrak{M}}(L, x)$. According the definition of the pseudo-Riemannian metrics $d$ on $G_{\mathfrak{M}}(F)$, this mapping is a local isometry and therefore, is a pseudo-Riemannian covering. The above proven isometricity of $p_{2}^{-1}(x)$ and $L_{0}$ implies Statement (i) of the theorem.

We consider an arbitrary leaf $\mathbb{L}=p_{1}^{-1}(L)$ of the induced foliation $\mathbb{F}$ on the graph $G_{\mathfrak{M}}(F)$ with the metrics $\left.d\right|_{\mathbb{L}}$. As it has been shown in Subsection 4.2, a pseudo-Riemannian manifold $(\mathbb{L}, d)$ is nondegenerately reducible and $\left.F^{(1)}\right|_{\mathbb{L}}$ and $\left.F^{(2)}\right|_{\mathbb{L}}$ its orthogonal parallel leaves. We stress that $\left.T F^{(1)}\right|_{\mathbb{L}}$ is 
an integrable Ehresmann connection for $\left.F^{(2)}\right|_{\mathbb{L}}$, and $\left.T F^{(2)}\right|_{\mathbb{L}}$ is an integrable Ehresmann connection for $\left.F^{(1)}\right|_{\mathbb{L}}$. Thus, $\left(\mathbb{L},\left.p_{1}\right|_{\mathbb{L}},\left.p_{1}\right|_{\mathbb{L}}, L, L\right)$ is a symmetric simple transversal bifibration in the sense [8].

We fix a point $z=\left(x,\left\{1_{x}\right\}, x\right) \in \mathbb{L}$. According (i), the group $\Psi \cong H_{\mathfrak{M}}(L, x)$ acts by isometries on a pseudo-Riemannian covering manifold $L_{0}$ as a group of covering transformations and this is why, a diagonal action $\Phi$ of the group $\Psi$ on a pseudo-Riemannian product $L_{0} \times L_{0}$ is well-defined by the rule $\psi\left(z_{1}, z_{2}\right)=\left(\psi\left(z_{1}\right), \psi\left(z_{2}\right)\right),\left(z_{1}, z_{2}\right) \in L_{0} \times L_{0}$. The action of $\Phi$ is free, properly discontinuous and preserves the structure of the product. The quotient manifold $\left(L_{0} \times L_{0}\right) / \Psi$ is well-defined with a pair of foliations $F_{1}, F_{2}$ covered by the product $L_{0} \times L_{0}$. Since $\Phi$ preserves the metrics of the pseudoRiemannian product on $L_{0} \times L_{0}$, then on $\left(L_{0} \times L_{0}\right) / \Psi$, a pseudo-Riemannian metrics is induced, and with respect to this metrics, the quotient mapping $L_{0} \times L_{0} \rightarrow\left(L_{0} \times L_{0}\right) / \Psi$ is a pseudo-Riemannian covering. At that, on $\left(L_{0} \times L_{0}\right) / \Psi$ a pair of parallel foliations $\left(F_{1}, F_{2}\right)$ is well-defined, whose leaves are covered by the leaves of trivial foliations of the product $L_{0} \times L_{0}$.

As it is known [8, Props. 5, 6], there exists a diffeomorphism

$$
\Theta: \mathbb{L} \rightarrow\left(L_{0} \times L_{0}\right) / \Psi
$$

being an isomorphism of both pairs of the foliations $\left.F^{(i)}\right|_{\mathbb{L}}$ and $F_{i}, i=1,2$ in the category of foliations Fol. It is easy to see that $L_{0} \times L_{0}$ is a common pseudo-Riemannian covering space for $\mathbb{L}$ and for $\left(L_{0} \times L_{0}\right) / \Psi$, therefore, $\Theta$ is a diffeomorphism being a local isometry, that is, $\Theta$ is an isometry and this completes the proof of Statement (ii) of Theorem 3 .

\section{Graphs of Parallel foliations}

\subsection{Criterion of existence of integrable Ehresmann connection.}

Definition 5. A pair of transversal foliations of additional dimensions $\left(F_{1}, F_{2}\right)$ on a manifold $M$ is called the a bifibration. true.

If $\left(F_{1}, F_{2}\right)$ is a bifibration on $M$, then at each point $x \in M$, the identity $T_{x} M=T_{x} F_{1} \oplus T_{x} F_{2}$ holds

Definition 6. Let $\left(F_{1}, F_{2}\right)$ be a bifibration on $M$ and $\kappa: \widehat{M} \rightarrow M$ be an universal covering mapping. If the conditions hold:

1) $\widehat{M}=M_{1} \times M_{2}$ is a product of simply-connected manifolds $M_{1}$ and $M_{2}$,

2) $\kappa^{*} F_{1}=\left\{M_{1} \times\left\{x_{2}\right\} \mid x_{2} \in M_{2}\right\}, \kappa^{*} F_{2}=\left\{x_{1} \times M_{2} \mid x_{1} \in M_{1}\right\}$,

then one says that the bifibration $\left(F_{1}, F_{2}\right)$ is covered by a product.

Employing the theorem by S. Kashiwabara [17, Thm. 2], it is easy to obtain the criterion of the existence of an integrable Ehresmann connection for a smooth foliation. We formulate this criterion in the following form convenient for us.

Theorem 5. A bifibration $\left(F_{1}, F_{2}\right)$ on a manifold $M$ is covered by a product if and only if the distribution $\mathrm{TF}_{2}$ is an integrable Ehresmann connection for the foliation $\left(M, F_{1}\right)$.

Let $\left(F_{1}, F_{2}\right)$ be a bifibration on the manifold $M$. The definition of Ehresmann connection implies that $T F_{2}$ is an Ehresmann connection for the foliation $\left(M, F_{1}\right)$ if and only if $T F_{1}$ is an Ehresmann connection for $\left(M, F_{2}\right)$.

6.2. Lemma. We shall employ the following statement, which is essentially of a local character.

Lemma 2. Let $\left(F, F^{\perp}\right)$ be mutually orthogonal foliations of additional dimensions on a pseudoRiemannian manifold $(M, g)$ and the induced metrics on the leaves of the foliations $(M, F)$ is nondegenerate. Then the following four statements are equivalent:

(1) the foliation $(M, F)$ is simultaneously pseudo-Riemannian and totally geodesic;

(2) both foliations $(M, F)$ and $\left(M, F^{\perp}\right)$ are totally geodesic;

(3) both foliations $(M, F)$ and $\left(M, F^{\perp}\right)$ are pseudo-Riemannian;

(4) both foliations $(M, F)$ and $\left(M, F^{\perp}\right)$ are parallel.

Proof. Assume that under the assumptions of the lemma $\operatorname{dim}(M)=n$ and $\operatorname{dim}(F)=q$, where $0<q<n$; at that, $\operatorname{dim}\left(F^{\perp}\right)=n-q$.

Let the foliation $(M, F)$ be simultaneously pseudo-Riemannian and totally geodesic. Since $(M, F)$ is a pseudo-Riemannian foliation, according [11, Thm. 1], the orthogonal $(n-q)$-dimensional distribution 
$\mathfrak{M}^{\perp}$ is totally geodesic. Since $\mathfrak{M}^{\perp}=T F^{\perp}$, this means that the foliation $\left(M, F^{\perp}\right)$ is totally geodesic. Hence, $(1) \Rightarrow(2)$.

Assume that both foliations $(M, F)$ and $\left(M, F^{\perp}\right)$ are totally geodesic. According [11, Thm. 1], both these foliations are pseudo-Riemannian, that is, $(2) \Rightarrow(3)$.

Let both foliations $(M, F)$ and $\left(M, F^{\perp}\right)$ are pseudo-Riemannian. As it is known, for each bifibration $\left(F, F^{\perp}\right)$, at each point $x \in M$, there exists a chart $(U, \varphi)$ such that $U=U_{1} \times U_{2}$, where $\left.F\right|_{U}=$ $\left\{U_{1} \times\left\{x_{2}\right\} \mid x_{2} \in U_{2}\right\}$ and $\left.F^{\perp}\right|_{U}=\left\{\left\{x_{1}\right\} \times U_{2} \mid x_{1} \in U_{1}\right\}$. Since the foliations $(M, F)$ and $\left(M, F^{\perp}\right)$ are pseudo-Riemannian, this implies the existence of pseudo-Riemannian metrics $g_{1}$ and $g_{2}$ on $U_{1}$ and $U_{2}$, respectively, such that the projections on the factors $U_{1} \times U_{2} \rightarrow U_{i}, i=1,2$, are pseudo-Riemannian submersions $\left(U_{1} \times U_{2}, g\right)$ on $\left(U_{i}, g_{i}\right)$. This means that $\left(U_{1} \times U_{2}, g\right)$ is a pseudo-Riemannian product of pseudo-Riemannian manifolds $\left(U_{1}, g_{1}\right)$ and $\left(U_{2}, g_{2}\right)$. Since $x$ is an arbitrary point in the manifold $M$, this yields that $(M, g)$ is a non-degenerate pseudo-Riemannian manifold with parallel foliations $(M, F)$ and $\left(M, F^{\perp}\right)$, see, for instance, [19]. Thus, we have proved that $(3) \Rightarrow(4)$.

Assume that $(M, F)$ and $\left(M, F^{\perp}\right)$ are parallel foliations on $(M, g)$. Since the tangential vectors to a geodesic curve forms a field of parallel translation, the definition of the parallel foliations imply that they are totally geodesic and this is why it follows from the proven implication $(2) \Rightarrow(3)$ that both foliations are pseudo-Riemannian. Thus, $(4) \Rightarrow(1)$.

\subsection{Holonomy groups of parallel foliations.}

Proposition 6. Let $F$ be a parallel foliation on a pseudo-Riemannian manifold $(M, g)$, the induced metrics be non-degenerate on the leaves and the distribution complementary with respect to the orthogonality is an Ehresmann connection for $F$. Then almost each leaf in the foliation $F$ has a trivial holonomy group.

Proof. The assumptions imply the existence a parallel foliation $F^{\perp}$ complementary to $F$ with respect to the orthogonality. Since $T F^{\perp}$ is an Ehresmann connection for $F$, it follows from Theorem 5 that the bifibration $\left(F, F^{\perp}\right)$ is covered by a product, that is, the universal covering for $M$ reads as $\kappa: M_{1} \times M_{2} \rightarrow M$ and $\kappa^{*} F=\left\{M_{1} \times\{z\} \mid z \in M_{2}\right\}$. We fix $x_{0} \in M$ and $\left(y_{0}, z_{0}\right) \in M_{1} \times M_{2}$, $p_{1}\left(y_{0}, z_{0}\right)=x_{0}$. By $L$ and $L^{\perp}$ we denote the leaves of the foliations $F$ and $F^{\perp}$ passing through $x_{0}$. We define $M_{1} \cong M_{1} \times\left\{z_{0}\right\}$ and $M_{2} \cong\left\{y_{0}\right\} \times M_{2}$. Let $g_{1}=\left.g\right|_{L}$ and $g_{2}=\left.g\right|_{L^{\perp}}$. Since $\left.\kappa\right|_{M_{1}}: M_{1} \rightarrow L$ and $\left.\kappa\right|_{M_{2}}: M_{2} \rightarrow L^{\perp}$ are universal covering mappings, the pseudo-Riemannina manifolds $\left(M_{1}, \kappa^{*} g_{1}\right)$ and $\left(M_{2}, \kappa^{*} g_{2}\right)$ are well-defined. Since locally $(M, g)$ is a product of pseudo-Riemannian manifolds induced on local leaves of foliations $F$ and $F^{\perp}$, the pseudo-Riemannian manifold $\left(M_{1} \times M_{2}, \kappa^{*} g\right)$ is a product of pseudo-Riemannian manifolds $\left(M_{1}, \kappa^{*} g_{1}\right)$ and $\left(M_{2}, \kappa^{*} g_{2}\right)$.

The fundamental group $\pi_{1}\left(M, x_{0}\right)$ acts on $M_{1} \times M_{2}$ as a group of covering transformations $G$ of the covering $\kappa$ preserving the structure of the product and a pseudo-Riemannian metrics $\kappa^{*} g$. This is why on $M_{2}$, a group of isometries $\Psi$ is induced and an epimorphism of the groups $\chi: G \rightarrow \Psi$ is well-defined. Since the action of the group $\Psi$ on $M_{2}$ is quasi-analytic, the holonomy group $\Gamma(L, x)$ of an arbitrary leaf $L=L(x)$ of the foliation $F$ is isomorphic to the stationary subgroup $\Psi_{z}$ of the group $\Psi$ at a point $z \in \operatorname{pr}\left(\kappa^{-1}(x)\right)$, where $p r: M_{1} \times M_{2} \rightarrow M_{2}$ is the canonical projection onto the second factor. Therefore, the leaf $L=L(x)$ possesses a trivial holonomy group if and only if the group $\Psi_{z}$ is trivial as $z \in \operatorname{pr}\left(\kappa^{-1}(x)\right)$.

Let fix $(\psi)$ be the set of fixed points of an isometry $\psi \in \Psi$. Let us prove that union of all leaves in the foliation $(M, F)$ with non-trivial holonomy groups has a zero measure in $M$. This is equivalent to the fact that the set $K=\bigcup_{\psi \in \Psi} f i x(\psi)$ has a zero measure in $M_{2}$.

We recall that a subset $N$ of a $m$-dimensional manifold has a zero measure if at each point there exists a chart $(U, f)$ of this manifold such that the subset $f(U \cap N) \subset \mathbb{R}^{m}$ has a zero measure in $\mathbb{R}^{m}$.

Let $\psi$ be an arbitrary element in $\Psi$ and $z$ be an arbitrary point in fix $(\psi)$. Since a pseudoRiemannian metrics defines a $G$-structure of the first order, there exists an isomorphism $\mu: \Psi_{z} \rightarrow D \Psi_{z}$, $\mu\left(\{\psi\}_{z}\right)=\psi_{* z}$, of the stationary subgroup $\Psi_{z}$ onto the linear group $D \Psi_{z}$ mapping each isometry $\psi \in \Psi_{z}$ into the differential $\psi_{* z}$ at the point $z$.

There exists a neighbourhood $W_{0}$ of the zero in $T_{z} M_{2}$ such that the exponential mapping $\left.E x p\right|_{W_{0}}$ : $W_{0} \rightarrow M_{2}$ is a diffeomorphism onto an open neighbourhood $W$ of the point $z$ in $M_{2}$. By the continuity of $\psi_{* z}$, there exists a neighbourhood $W_{0}^{\prime}$ of the zero in $T_{z} M_{2}$ obeying $\psi_{* z}\left(W_{0}^{\prime}\right) \subset W_{0}$. Let $W^{\prime}=$ 
$\operatorname{Exp}\left(W_{0}^{\prime}\right)$. Since $\psi$ is an isometry, it satisfies the identity

$$
\left.\operatorname{Exp} \circ \psi_{* z}\right|_{W_{0}^{\prime}}=\left.\psi \circ \operatorname{Exp}\right|_{W_{0}^{\prime}}
$$

and therefore, $\operatorname{Exp}^{-1}\left(f i x(\psi) \cap W^{\prime}\right)=f i x\left(\psi_{* z}\right) \cap W_{0}^{\prime}$. Since $\psi_{* z}$ is a non-trivial linear mapping of the vector space $T_{z} M_{2}$, the set $f i x\left(\psi_{* z}\right)$ is a proper subspace in $T_{z} M_{2}$ and this is why set $f i x\left(\psi_{* z}\right) \cap W_{0}^{\prime}$ has a zero measure in $T_{z} M_{2} \cong \mathbb{R}^{q}$. We observe that $\left(W^{\prime},\left.\operatorname{Exp}^{-1}\right|_{W^{\prime}}\right)$ can be treated as a chart at the point $z$ in the manifold $M_{2}$. Therefore, the set $f i x(\psi)$ has a zero measure in $M_{2}$. Since the group $\Psi$ is at most countable, this implies that the set $K$ has also a zero measure in $M_{2}$.

6.4. Proof of Theorem 4. Assume that $(M, g)$ is a non-degenerate reducible pseudo-Riemannian manifold, while $F$ and $F^{\perp}$ are its parallel foliations of additional dimension and $\mathfrak{M}=T F^{\perp}$ is an Ehresmann connection for the foliation $(M, F)$. By Lemma 2 , the foliations $F$ and $F^{\perp}$ are simultaneously totally geodesic and pseudo-Riemannian. This is why the holonomy pseudo-group of the foliation $(M, F)$ is formed by local isometries and is quasi-analytic. Hence, Proposition 1 implies that the graph $G(F)$ is Hausdorff. According Theorem 1, the graphs $G(F)$ and $G_{\mathfrak{M}}(F)$ are canonically isomorphic and are identified and this proves Statement 1.

According [11, Thm. 2], the induced foliation $(G(F), \mathbb{F})$ is also pseudo-Riemannian and Statement 2 follows Proposition 6 and Statements 2, 3 of Theorem 3 .

Let us show that the distribution $\mathfrak{N}$ is integrable. For each $X \in \mathfrak{X}_{\mathfrak{N}}(G(F))$, the properties of the differential implies $p_{i *}(X) \in \mathfrak{X}_{\mathfrak{M}}(M)$ as $i=1,2$. Taking this into consideration, by the integrability of $\mathfrak{M}$ we have

$$
p_{1 *}([X, Y])=\left[p_{1 *}(X), p_{1 *}(Y)\right] \in \mathfrak{X}_{\mathfrak{M}}(M)
$$

and

$$
p_{2 *}([X, Y])=\left[p_{1 *}(X), p_{2 *}(Y)\right] \in \mathfrak{X}_{\mathfrak{M}}(M) .
$$

Hence, in view of the identity $\mathfrak{N}=p_{1}^{*} \mathfrak{M} \cap p_{2}^{*} \mathfrak{M}$, we obtain $[X, Y] \in \mathfrak{X}_{\mathfrak{N}}(G(F)$ ). By the Frobenius theorem, the distribution $\mathfrak{N}$ is integrable and defines a foliation, which we denote by $F^{\mathfrak{N}}$. It follows from Theorem 2 that the foliations $F^{\mathfrak{N}}$ and $\mathbb{F}$ are totally geodesic. According Lemma 2 this is equivalent to the fact that $\left(F^{\mathfrak{N}}, \mathbb{F}\right)$ is a pair of parallel foliations complementary with respect to the orthogonality.

Let us show that the distribution $\mathfrak{M}^{(2)}$ is also integrable. We take arbitrary vector fields $X, Y$ tangential to $\mathfrak{M}^{(2)}$. Let $Z:=[X, Y]$. Since $\mathfrak{M}^{(2)}=T F^{(1)} \oplus \mathfrak{N}=p_{1}^{*} \mathfrak{M}$, thanks to the integrability of the distribution $\mathfrak{M}=T F^{\perp}$, the chain of identities holds:

$$
p_{1 *}(Z)=p_{1 *}([X, Y])=\left[p_{1 *}(X), \quad p_{1 *}(Y)\right] \in \mathfrak{X}_{\mathfrak{M}}(M),
$$

and this is why it is necessary that $Z \in \mathfrak{X}_{\mathfrak{M}(2)}(G(M))$. According Frobenius theorem, the distribution $\mathfrak{M}^{(2)}$ is integrable. We denote by $\mathcal{F}^{(2)}$ the foliation, for which $\mathfrak{M}^{(2)}=T \mathcal{F}^{(2)}$. By the assumptions, the foliations $(M, F)$ and $\left(M, F^{\perp}\right)$ are parallel and this is why it follows from Lemma 2 that the foliation $(M, F)$ is totally geodesic. By Theorem 2 , the total geodesicity of the foliation $(M, F)$ implies the same for the foliation $\left(G(F), F^{(2)}\right)$, and according [11, Thm. 2], the pseudo-Riemannian property of $(M, F)$ implies the same for the foliation $\left(G(F), F^{(2)}\right)$. This is why, thanks to Lemma 2 , the foliations $F^{(2)}$ and $\mathcal{F}^{(2)}$ on the graph $(G(F), d)$ complementary with respect to the orthogonality are parallel. In the same way we prove that the pair of complementary with respect to the orthogonality foliations $\left(F^{(1)}, \mathcal{F}^{(1)}\right)$ are parallel. This completes the proof of Statements 2 and 3.

We consider the universal covering mapping $f: \widetilde{G(F)} \rightarrow G(F)$. Since $\mathfrak{N}=T F^{\mathfrak{N}}$ is an integrable Ehresmann connection for the foliation $(G(M), \mathbb{F})$, by Theorem $5, \widetilde{G(F)}=\widetilde{L^{\mathfrak{N}}} \times \widetilde{\mathbb{L}}$ is a product of simply-connected manifolds and $f^{*} F^{\mathfrak{N}}=\left\{\widetilde{L^{\mathfrak{N}}} \times\{v\} \mid v \in \widetilde{\mathbb{L}}\right\}$ and $f^{*} \mathbb{F}=\left\{\{u\} \times \widetilde{\mathbb{L}} \mid u \in \widetilde{L^{\mathfrak{N}}}\right\}$. It follows from Theorem 2 that $\widetilde{\mathbb{L}} \cong \widetilde{L_{0}} \times \widetilde{L_{0}}$, where $\left.f\right|_{\widetilde{L_{0}}}: \widetilde{L_{0}} \rightarrow L_{0}$ is an universal covering mapping for $L_{0}$. Thus, $\widetilde{G(F)} \cong \widetilde{L^{\mathfrak{N}}} \times \widetilde{L_{0}} \times \widetilde{L_{0}}$. Since the bifibration $\left(\mathcal{F}^{(2)}, F^{(2)}\right)$ is covered by a product $\left(\widetilde{L^{\mathfrak{N}}} \times \widetilde{L_{0}}\right) \times \widetilde{L_{0}}$, according Theorem 5, $T F^{(2)}$ is an integrable Ehresmann connection for the foliation $\left(G(F), \mathcal{F}^{(2)}\right)$. And vice versa, $T \mathcal{F}^{(2)}$ is an integrable Ehresmann connection for $\left(G(F), F^{(2))}\right.$. In the same way, $T F^{(1)}$ is an integrable Ehresmann connection for $\left(G(F), \mathcal{F}^{(1)}\right)$ and vice versa. This completes the proof of Statement 3 of the theorem. 


\section{Two ClASSES OF STUDIED FOLIATIONS}

7.1. Proof of Proposition 4. The family of piece-wise smooth geodesic curves of each pseudoRiemannian manifold form a system of paths in the sense of [18]. Therefore, on each leaf $\left(L_{\alpha}, g\right)$ of the foliation $(M, F)$ a system of paths is well-defined. By the completeness of the induced metrics on the leaves, the affine parameter of each geodesic curve lying in a leaf varies on the entire real line. This means that the mentioned system of paths is complete. According Lemma 1 and [16, Prop. 2.7], thanks to the total geodesicity of the foliation $(M, F)$, for each integral curve $\sigma$ of the distribution $\mathfrak{M}$, a local horizontal holonomic diffeomorphism $\Phi_{\sigma}: U_{0} \rightarrow U_{1}$ is an isometry. Therefore, $\Phi_{\sigma}$ maps a geodesic curve into a geodesic curve keeping the parameter. This means that the foliation $(M, F)$ is $\mathfrak{M}$-conformed with the systems of paths on the leaves. Hence, it follows from [18, Thm. 6.1] that $\mathfrak{M}$ is an Ehresmann connection for the foliation $(M, F)$.

\subsection{Suspended foliations over pseudo-Riemannian manifolds.}

Suspended foliations. Let $\left(B, g^{B}\right)$ be an arbitrary $m$-dimensional pseudo-Riemannian manifold and $T$ be an arbitrary smooth $q$-dimensional manifold. Suppose that we are given a homomorphism $\rho: G \rightarrow$ $\operatorname{Diff}(T)$ of the group $G=\pi_{1}(B, b)$ into the group $\operatorname{Diff}(T)$ of diffeomorphisms of the manifold $T$. Let the group $G$ act from the right as a group of covering transformations on the universal covering space $\widehat{B}$. Then the identity $f(x, t, g)=\left(x \cdot g, \rho\left(g^{-1}\right)(t)\right)$, where $(x, t, g) \in \widehat{B} \times T \times G$, defines a right action of the group $G$ on the product of the manifolds $\widehat{B} \times T$. A quotient mapping $f: \widehat{B} \times T \rightarrow M$ onto the quotient manifold $M:=(\widehat{B} \times T) / G$ induces a smooth foliation $F=\{f(\widehat{B} \times\{v\}) \mid v \in T\}$ on $M$, which is called suspended and is denoted by $(M, F)=\operatorname{Sus}(T, B, \rho)$. The projection $p: M=(\widehat{B} \times T) / G \rightarrow B=\widehat{B} / G$ forms a locally trivial fibration, which is called associated. The group $\Psi=\rho\left(\pi_{1}(B, b)\right)$ is called the structural group of the suspended foliation $(M, F)$.

7.3. Proof of Proposition 3. Let $p: M \rightarrow B$ be the associated fibration and $\mathfrak{M}$ be a distribution formed by tangential spaces to its fibres. Each vector field $X$ on $M$ is uniquely represented as $X=X^{F}+X^{\mathfrak{M}}$, where $X^{F} \in \mathfrak{X}_{F}(M), X^{\mathfrak{M}} \in \mathfrak{X}_{\mathfrak{M}}(M)$. Let $g^{M}$ be a pseudo-Riemannian metrics $M$ non-degenerate on the leaves of the foliation $(M, F)$. A mentioned metrics $g^{M}$ exists since as $g^{M}$ we can take an arbitrary Riemannian metrics. Then the identity

$$
g(X, Y):=\left(p^{*} g^{B}\right)\left(X^{F}, Y^{F}\right)+g^{M}\left(X^{\mathfrak{M}}, Y^{\mathfrak{M}}\right) \quad \text { for all } \quad X, Y \in \mathfrak{X}(M)
$$

defines a pseudo-Riemannian metrics $g$ on the manifold $M$. The definition of the metrics $g$ implies that $p: M \rightarrow B$ is a pseudo-Riemannian submersion $(M, g)$ on $\left(B, g^{B}\right)$. Therefore, local horizontal holonomic diffeomorphisms of the foliation $F$ are isometries and this is why, according Proposition 5 , $F$ is a totally geodesics foliation on a pseudo-Riemannian manifold $(M, g)$. By the properties of the suspended manifold, the restriction $\left.p\right|_{L_{\alpha}}$ of the projection $p$ on an arbitrary leaf $L_{\alpha}$ of the foliation $F$ is a covering mapping onto the base $B$ and therefore, $\left.p\right|_{L_{\alpha}}: L_{\alpha} \rightarrow B$ is a pseudo-Riemannian covering mapping. This implies that the leaves $L_{\alpha}$ equipped with the induced pseudo-Riemannian metrics are complete pseudo-Riemannian manifolds if and only if $\left(B, g^{B}\right)$ is a complete pseudo-Riemannian manifold.

The completeness of the metrics $g^{B}$ is not assumed. Thus, we have constructed a totally geodesic foliation $(M, F)$ on the pseudo-Riemannian manifold $(M, g)$ with induced pseudo-Riemannian metrics on the leaves, whose orthogonal $q$-dimensional distribution $\mathfrak{M}$ is an integrable Ehresmann connection for this foliation.

The proof of Statements 1)-3) is complete.

We stress that the graph of the foliation $G(F)$ is generally speaking not Hausdorff. Since the holonomy pseudo-group $\mathcal{H}(F)$ of the foliation $F$ is determined by the transformations in the group $\Psi:=\rho\left(\pi_{1}(B, b)\right) \subset \operatorname{Diff}(T)$, by applying Proposition 1 we conclude that the graph $G(F)$ is Hausdorff if and only if the group $\Psi$ acts quasianalytically on $T$. This completes the proof of Statement 4 ).

Thus, the foliations obtained by a suspension of a homomorphism of the fundamental group of a pseudo-Riemannian manifold belong to the studied class of the foliations.

Remark 4. In terms of the above introduced notations, the graph $G_{\mathfrak{M}}(F)$ of the suspended foliation $(M, F)$ is a Hausdorff smooth $(2 m+q)$-dimensional manifold with a pseudo-Riemannian metrics $d$. The fibres of the canonical projections $p_{1}$ and $p_{2}$ are totally geodesic $m$-dimensional submanifolds in 
$\left(G_{\mathfrak{M}}(F), d\right)$ isometric to arbitrary leaf $\left(L_{0}, d\right)$ with the trivial $\mathfrak{M}$-holonomy group of the foliation $(M, F)$ once it exists. A q-dimensional distributioin $\mathfrak{N}$ orthogonal to the induced totally geodesic foliation $\mathbb{F}$ is integrable and is tangent to some pseudo-Riemannian foliation.

\section{BIBLIOGRAPHY}

1. H.E. Winkelnkemper. The graph of a foliation // Ann. Global Anal. Geom. 1:3, 51-75 (1993).

2. I. Tamura. Topology of foliations: An Introduction. Transl. Math. Monogr. 97. Amer. Math. Soc., Providence, RI (1992).

3. A. Connes. Non-commutative geometry. Academic Press, Boston (1994).

4. R. Blumenthal, J. Hebda. Ehresmann connections for foliations // Indiana Univ. Math. J. 33:4, 597-611 (1984).

5. R. Blumenthal, J. Hebda. Complementary distributions which preserve the leaf geometry and applications to totally geodesic foliations// Quarterly J. Math. Oxford Ser. (2). 35:4, 383-392 (1984).

6. N.I. Zhukova. The graph of a foliation with an Ehresmann connection and the stability of leaves // Izv. VUZov. Matem. 2, 79-81 (1994). [Russian Math. Iz. VUZ. 38:2, 76-79 (1994).]

7. N.I. Zhukova. Properties of the graphs of Ehresmann foliations // Vestn. NNGU. Ser. Mat. 1, 73-87 (2004). (in Russian).

8. N.I. Zhukova. Singular foliations with Ehresmann connections and their holonomy groupoids // Banach Center Publ. 76, 471-490 (2007).

9. N.I. Zhukova. Local and global stability of compact leaves and foliations// J. Math. Phys. Anal. Geom. 9:3, 400-420 (2013).

10. R. A. Wolak. The graph of a totally geodesic foliation// Ann. Polonici Mathematici. 60:3, 241-247 (1995).

11. A.Yu. Dolgonosova, N.I. Zhukova. Pseudo-Riemannian foliations and their graphs // Lobachevskii J. Math. 39:1, 54-64 (2018).

12. B. O'Neill. Semi-Riemannian geometry with applications to relativity. Academic Press, New York (1983).

13. A. Gray. Pseudo-Riemannian almost product manifolds and submersions // J. Math. Mech. 16, 715-737 (1967).

14. G. Baditoiu. Classification of Pseudo-Riemannian submersions with totally geodesic fibres from pseudo-hyperbolic spaces// Proc. London Math. Soc. 105:6, 1315-1338 (2012).

15. Sh. Kobayashi, K. Nomizu. Foundations of differential geometry. Vol. I. John Wiley \& Sons, New York (1963).

16. K. Yokumoto. Mutual exclusiveness along spacelike, timelike, and lightlike leaves in totally geodesic foliations of lightlike complete Lorentzian two-dimensional tori // Hokkaido Math. J. 31:3, 643-663 (2002).

17. S. Kashiwabara. The decomposition of a differentiable manifolds and its applications // Tohoku Math. J. (2). 11:1, 43-53 (1959).

18. N.I. Zhukova. Foliations that are compatible with systems of paths // Izv. VUZov. Matem. 7, 5-13 (1989). [Soviet Math. Iz. VUZ. 33:7, 5-15 (1989).]

19. H. Wu. On the de Rham decomposition theorem// Illinois J. Math. 8:2, 291-311 (1964).

Nina Ivanovna Zhukova,

National Research University

Higher School of Economics,

Bol'shaya Pecherskaya str. 25/12,

603155, Nizhny Novgorod, Russia

E-mail: nzhukova@hse.ru 